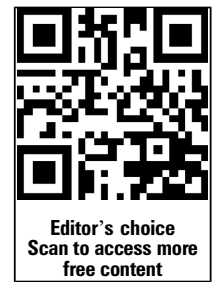

${ }^{1}$ Hôpital Bichat, Service de Cardiologie, Paris, France ${ }^{2}$ Centre National de Référence pour le syndrome de Marfan et apparentés, Hôpital Bichat, AP-HP, Paris, France

${ }^{3}$ Division of Cardiovascular Diseases, Mayo Clinic Rochester, Minnesota, USA ${ }^{4}$ Université Paris 7, Paris, France

\section{Correspondence to} Dr Hector I Michelena, Division of Cardiovascular Diseases, Mayo Clinic, 200 First Street SW, Rochester, MN 55905 , USA

michelena.hector@mayo.edu

Received 3 September 2013 Revised 18 October 2013 Accepted 21 October 2013 Published Online First 19 November 2013

\section{SLinked}

- http://dx.doi.org/10.1136 heartjnl-2013-305004

To cite: Detaint $D$ Michelena HI, Nkomo VT, et al. Heart 2014;100: 126-134.

\title{
Aortic dilatation patterns and rates in adults with bicuspid aortic valves: a comparative study with Marfan syndrome and degenerative aortopathy
}

\author{
Delphine Detaint, ${ }^{1,2}$ Hector I Michelena, ${ }^{3}$ Vuyisile T Nkomo, ${ }^{3}$ Alec Vahanian, ${ }^{1,4}$ \\ Guillaume Jondeau, ${ }^{1,2,4}$ Maurice Enriquez Sarano ${ }^{3}$
}

\begin{abstract}
Background Bicuspid aortic valve (BAV) is related to aortic dilatation, but patterns/rates are conflicting with no comparison among aneurysms of different aetiology. We sought to define ascending aorta dilatation patterns/ progression rates in BAV versus other aortopathies (Marfan syndrome (MFS), degenerative aortopathy (DA)). Design and setting Retrospective, observational study. Aortic dilatation progression was evaluated in two tertiary care centres (US and European) by repeated echocardiography $\geq 2$ years apart in adults with BAV $(n=353)$, matched to MFS $(n=50)$ and DA $(n=51)$ for gender, blood pressure, and minimum follow-up time. Results At baseline, ascending aortic dilatation was present in $87 \%$ of BAV cases: tubular ascending aorta in $60 \%$ (irrespective of BAV morphology), and Valsalva sinuses dilatation in $27 \%$ (independently linked to typical BAV morphology and male gender $(p=0.0001))$.
\end{abstract} After $3.6 \pm 1.2$ years, the aortic dilatation rate in BAV was higher than expected for the population for all aortic levels $(p=0.005)$ and was maximal at the tubular ascending aorta for BAV $(0.42 \pm 0.6 \mathrm{~mm} /$ year) and DA $(0.20 \pm 0.3 \mathrm{~mm} / \mathrm{year})$, and was maximal at the Valsalva sinuses for MFS $(0.49 \pm 0.5 \mathrm{~mm} /$ year). Maximal aortic dilatation rate was similar between BAV and MFS $(p>0.40)$ and lower in DA ( $p=0.02)$ but was heterogeneous in BAV, with $43 \%$ of BAV not progressing (vs $20 \%$ of MFS, $p=0.01$ ). Aortic dilatation rate was not proportionally related to baseline aortic size or BAV type (all models $p>0.40$ ).

Conclusions In patients with BAV, tubular ascending aorta dilatation is the most common pattern and exhibits the fastest growing rate, irrespective of valve morphology and function. Dilatation of the Valsalva sinuses is less common and associated with typical BAV morphology and male gender. Aortic dilatation progresses equally fast in BAV (tubular segment) and MFS (Valsalva sinuses), but a significantly higher proportion of BAV patients does not progress at all, irrespective of BAV type. Baseline aortic diameter does not proportionally predict progression rate; systematic follow-up is therefore warranted in patients with BAV.

\section{INTRODUCTION}

Bicuspid aortic valve (BAV) is a common congenital abnormality with a prevalence of $1.3 \% .{ }^{1} \mathrm{BAV}$ is associated with endocarditis ${ }^{2}$ and premature aortic valve dysfunction. ${ }^{3}{ }^{4}$ However, BAV is not just a valvular disorder but also an aortopathy, with aortic dilatation and risk of aortic dissection and rupture. ${ }^{5}$ Valvular dysfunction may play a role in the development of aortic abnormalities, but aortic dilatation is often out of proportion to the degree of valvular dysfunction, ${ }^{6}{ }^{7}$ suggesting an intrinsic aortic tissue abnormality. Indeed, BAV studies have reported structural abnormalities of thoracic aortic tissue, including decreased fibrillin, elastin fragmentation, matrix disruption, and apoptosis. ${ }^{8} 9$ These similarities to Marfan syndrome (MFS) led the American College of Cardiology/American Heart Association to recommend aggressive elective aortic surgery for BAV patients with aortic dilatation or sooner as possible for 'rapid progressors'. ${ }^{10}$ However, there are considerable uncertainties. Recent studies suggest that in BAV patients the risk of aortic catastrophes, although higher than in the general population, remains low. ${ }^{4}$ Additionally, reports of aortic dilatation progression involved limited sample sizes and paediatric populations, ${ }^{11}$ 12 and resulted in discordant progression rates ${ }^{13-15}$ with variable association with BAV function and morphology. Additionally, patterns of aortic dilatation with BAV were variably reported as predominantly involving the tubular ascending aorta (AA), ${ }^{16}$ the Valsalva sinuses and proximal $\mathrm{AA}^{6}{ }^{6}$ or as balanced, ${ }^{7}$ and were not compared to those of MFS. The controversies regarding aortic imaging similarities and differences between various types of aortopathies (BAV, MFS, and degenerative aortopathy (DA)) in regard to progression rates and patterns of aortic dilatation are unresolved. ${ }^{17}$ To address these controversies, we studied a large population of patients with BAV who were followed for at least 2 years at two large European and US centres, analysed aortic dilatation patterns, rates and their determinants, and as a secondary aim, compared BAV to other aortopathies.

\section{METHODS}

The study was approved by the institutional review boards for each institution. We identified patients in two tertiary referral centres (Mayo Clinic Rochester, Minnesota, USA, and Bichat Hospital, Paris, France) with the conditions listed below, who had echocardiograms between 2003 and 2008 and the latest follow-up echocardiogram performed at least 2 years later. Three different patients groups were assembled:

BAV patients: Adults (age $\geq 18$ years) with BAV identified by echocardiography were included. Exclusion criteria were previous cardiac surgery 
(except for aortic coarctation repair) or aortic dissection or the diagnoses of MFS or other severe congenital heart defect. No exclusions were made on the basis of associated valvular dysfunction.

$D A$ patients: Adults were included if they had a tricuspid aortic valve with dilatation of the sinuses or AA (diameter $>40 \mathrm{~mm}$ ) determined by echocardiogram. Exclusion criteria were previous cardiac surgery, aortic dissection, MFS, or severe congenital heart defect. No exclusions were made on the basis of associated valvular dysfunction. A total of 51 patients were matched to BAV patients for gender, systolic blood pressure at baseline, and timing of minimum follow-up echocardiography.

MFS patients: Adults were included from the French Marfan and Associated Disease Centre, provided they fulfilled international criteria ${ }^{18}$ including a mutation in the FBN1 gene and had a tricuspid aortic valve at echocardiography. Exclusion criteria were previous cardiac surgery, aortic dissection, and severe congenital heart defect. No exclusions were made on the basis of associated valvular dysfunction. A total of 50 patients were matched to patients with BAV for gender, systolic blood pressure at baseline, and timing of minimum follow-up echocardiography.

\section{Echocardiography}

All patients underwent comprehensive transthoracic echocardiography evaluation at baseline and at follow-up. All echocardiograms were prospectively reviewed blindly of diagnosis by an experienced observer (DD) to ensure homogeneity in the aorta measurements in both centres. Left ventricular ejection fraction was assessed by $2 \mathrm{D}$ echocardiography ${ }^{19}$ including visual estimation. ${ }^{20}$ Diagnosis of BAV was based on parasternal short axis imaging of the aortic valve using either transthoracic echocardiography or transoesophageal echocardiography at $45^{\circ}$, requiring the presence of only two commissures delimiting only two aortic cusps. Bicuspid valves were classified as typical (anterior and posterior cusps) when the commissures were at 4-10, 5-11 or 3-9 o'clock, and atypical (right and left cusps) when the commissures were at 1-7 or 12-6 o'clock (figure 1A). The presence of a raphe was recorded. Hence, four main types were identified; typical with and without raphe and atypical with and without raphe. ${ }^{3} 16$

Aortic valve function was evaluated using Doppler methods: aortic stenosis severity was assessed by the transaortic mean gradient and the aortic valve area calculated by the continuity equation. Aortic stenosis was defined as severe when the aortic valve area (AVA) was $\leq 1 \mathrm{~cm}^{2}$ or mean gradient $\geq 40 \mathrm{~mm} \mathrm{Hg}$, and as moderate when the AVA was $1-1.5 \mathrm{~cm}^{2}$ and mean gradient $25-40 \mathrm{~mm} \mathrm{Hg} .{ }^{10}$ Measurements of the aortic annulus were performed in early systole, parasternal long axis view zoomed on the left ventricular outflow tract, inner edge to inner edge, at insertion points of the aortic cusps. Aortic regurgitation (AR) evaluation ${ }^{21}$ was based on American Society of Echocardiography criteria, with classification as $\leq$ mild, moderate, and >moderate using colour Doppler jet width and extension, vena contracta width measurement and, when possible, quantitative measurements using the proximal isovelocity surface area method. The aetiology of AR was classified as: cusp prolapse, cusp degeneration (cusp thickening and restriction), predominant annular enlargement, or indeterminate.

All measurements of the aorta were performed by the same investigator (DD) according to recommendations and on the QRS complex of the ECG. ${ }^{22} 23$ The dimensions of the Valsalva sinuses were measured perpendicular to the right and left (or non-) aortic sinuses. The sinotubular junction (STJ) was measured where the aortic sinuses met the tubular aorta. The AA was measured approximately $2 \mathrm{~cm}$ distal to the STJ. To verify the validity of the echocardiographic measurements of the aortic dimensions, random cases were re-measured by a second observer and the echocardiographic measurements compared to clinically indicated MRI measurements performed during the same episode of care in 42 patients.

Three aortic phenotypes were defined for all groups: (1) normal shape: Valsalva sinuses $<37 \mathrm{~mm}$ and AA $<$ Valsalva; (2) predominant dilatation of the Valsalva sinuses (Valsalva $\geq 37 \mathrm{~mm}$ and Valsalva $>A A$ ); (3) predominant dilatation of the AA (AA $\geq 37 \mathrm{~mm}$ and $\mathrm{AA}>$ Valsalva) (figure $1 \mathrm{~B}$ ). Measurements were performed at baseline and at follow-up. Yearly dilatation rates were calculated as: [diameter 2-diameter 1]/time interval in years.

Expected diameters of the sinuses of Valsalva and STJ at baseline and follow-up were calculated from Roman's equations ${ }^{23}$ using age and the body surface area (BSA) of the BAV population, allowing for the calculation of an expected dilatation rate. Patients were identified as 'rapid progressors' if the dilatation rate of one aortic segment was within the upper quartile for that aortic segment (annulus, Valsalva sinuses, STJ, and AA) and as 'non-progressors' if the dilatation rate of one aortic segment was in the lower quartile.

\section{Statistics}

Data are presented as mean $\pm \mathrm{SD}$ or percentage as appropriate.

Baseline and follow-up values were compared using paired t test and the observed dilatation rate was compared to the expected dilatation rate using Student $t$ test. Comparisons between BAV, MFS, and DA used one-way analysis of variance (ANOVA) and nonparametric Wilcoxon test (in cases of significance, two by two comparisons were tested using Student $t$ test and post-hoc Tukey analysis). Among patients with BAV, the aortic patterns and subtypes of BAV were compared using ANOVA. To define an independent association, multivariate analyses were performed adjusting for significant univariable predictors. Univariable and multivariate logistic regression models were constructed to identify predictors of rapid progression. Rapid progressors (ie, dilatation rate within the upper quartile for each aortic segment) were thus defined as $\geq 0.2 \mathrm{~mm} /$ year, $\geq 0.34 \mathrm{~mm} /$ year, $\geq 0.4 \mathrm{~mm} /$ year, $\geq 0.6 \mathrm{~mm} /$ year, for annulus, sinuses of Valsalva, STJ, and AA, respectively. Non-progressors (ie, dilatation rate within the lower quartile) were defined as $\leq 0 \mathrm{~mm} /$ year for all levels. Candidate variables were age, gender, BSA, blood pressure, heart rate, aortic stenosis at baseline, AR at baseline, type of aortic dilatation, and type of BAV. Odd ratios of rapid progression were calculated. Avalue of $\mathrm{p}<0.05$ was considered significant.

\section{RESULTS}

\section{Baseline characteristics in BAV patients}

Baseline characteristics are summarised in the upper two thirds of table 1. We enrolled 353 adults with BAV (male 72\%) with baseline and follow-up echocardiograms separated by 3.6 \pm 1.2 years (1271 patient-years). Mean age was $48 \pm 15$ years and mean BSA $1.98 \pm 0.2 \mathrm{~m}^{2}$. BAV was typical with raphe in 204 (58\%), typical without raphe in 57 (16\%), atypical without raphe in $56(16 \%)$, and atypical with raphe in $29(8 \%)$. In seven patients (2\%), while certainty on BAV was confirmed, the typing was uncertain (figure 2, panel A, grey slice). Inter-observer echocardiographic variability was non-significant and in the 42 patients with MRI during the same episode of care, measurements were similar to echocardiography for Valsalva sinuses (36.7 vs $36.5 \mathrm{~mm}, \mathrm{p}=0.62)$ and $\mathrm{AA}(35.6$ vs $35.9 \mathrm{~mm}$, $\mathrm{p}=0.70)$.

AR was observed in $246(70 \%)$ BAV patients and was mild in 123 , moderate in 117 , and severe in six cases at baseline. Mean 

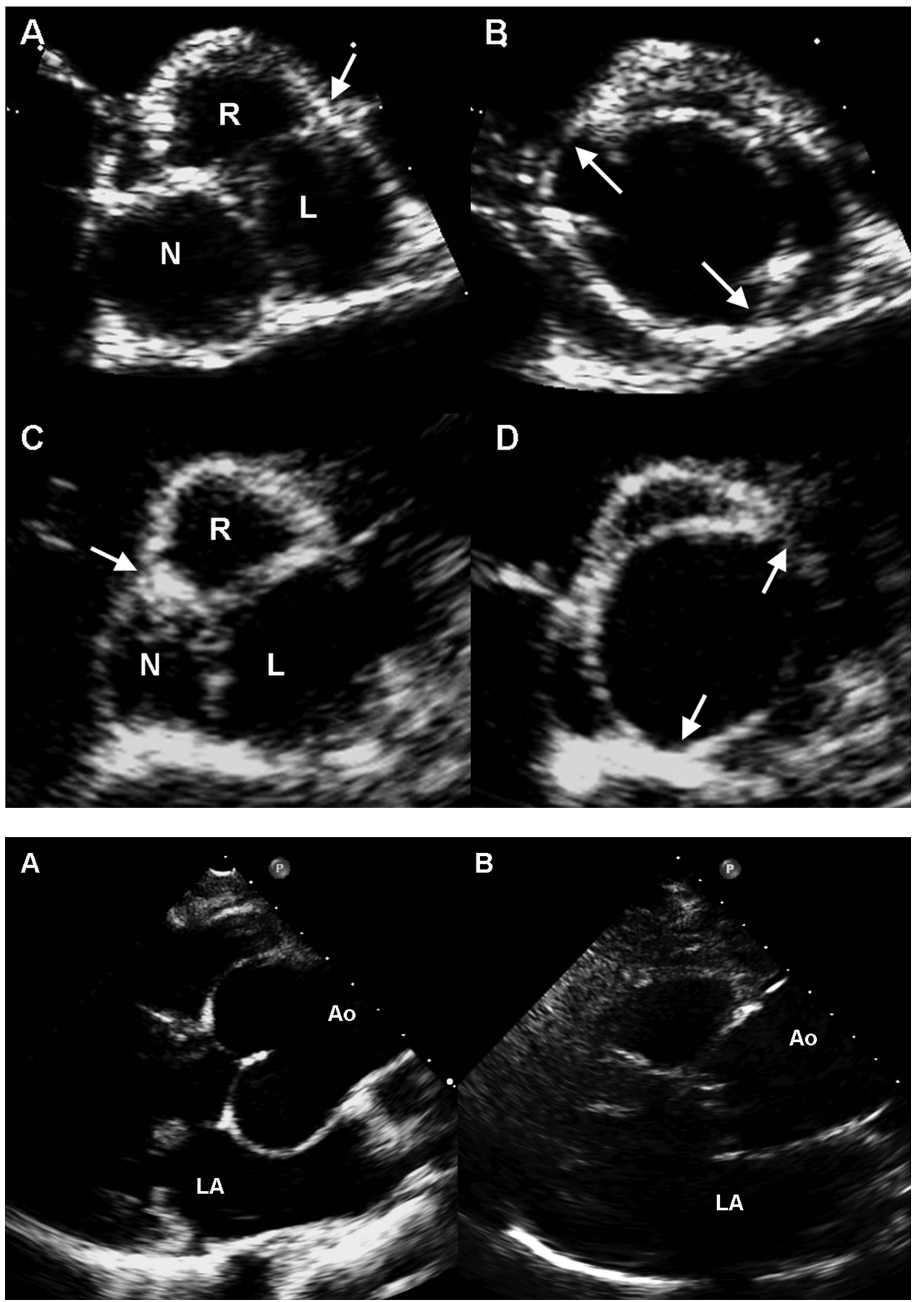

Figure 1 (A) Basic morphologies of bicuspid aortic valve (BAV). Panel A shows a typical BAV in diastole with a small raphe (arrow) between the right (R) and left (L) coronary cusps. In systole (panel B), commissures are located at 10 and 4 o'clock (arrows). Panel C depicts an atypical BAV in diastole with a prominent raphe (arrow) between the right (R) and non-coronary (N) cusps. In systole (panel D), commissures are located at 1 and 7 o'clock (arrows). (B) Aortic dilatation phenotypes. Panel A depicts a patient with Marfan syndrome and predominant dilatation of the sinuses of Valsalva. Panel B depicts a patient with BAV and predominant dilatation of the tubular ascending aorta. LA, left atrium; Ao, ascending aorta.

vena contracta was $2.3 \pm 2.2 \mathrm{~mm}$. AR was due to cusp prolapse in $37 \%$, degenerative disease in $24 \%$, predominant annular enlargement in 24\%, and indeterminate in $15 \%$. Significant aortic stenosis was observed in 51 (14\%) patients (moderate in 44 , severe in seven).

Comparison with other aortopathies at baseline

By design, compared to BAV patients, the group of 51 DA patients and 50 MFS patients were similar for gender, systolic blood pressure (all $\mathrm{p}=\mathrm{NS}$ ), and minimum follow-up time. Also,
BSA was similar between groups $(\mathrm{p}=0.59)$ but, as expected, their ages differed, with the youngest patients in the MFS group and the oldest in the DA group $(p<0.0001$, table 1$)$. $\mathrm{AR} \geq$ moderate was more frequent in $\mathrm{BAV}$ patients versus $\mathrm{DA}$ and MFS patients $(\mathrm{p}<0.0001)$, despite similar annular diameters and smaller Valsalva sinuses in BAV (table 1).

Aortic phenotype and BAV morphology/function at baseline Comparisons between aortic phenotype and BAV morphology/ function at baseline are presented in table 2. The most common 
Table 1 Baseline aortic diameters and yearly aortic dilatation rates in patients with BAV, DA, and MFS

\begin{tabular}{|c|c|c|c|c|}
\hline & $\begin{array}{l}\text { BAV } \\
N=353\end{array}$ & $\begin{array}{l}D A \\
N=51\end{array}$ & $\begin{array}{l}\text { MFS } \\
N=50\end{array}$ & p Value \\
\hline Age, year & $48 \pm 15$ & $71 \pm 11$ & $35 \pm 11$ & $<0.0001$ \\
\hline Gender, male $n(\%)$ & $254(72 \%)$ & $40(78 \%)$ & $36(72 \%)$ & 0.60 \\
\hline Systolic BP mm Hg (baseline) & $124 \pm 17$ & $124 \pm 16$ & $128 \pm 19$ & 0.59 \\
\hline Ejection fraction, $\%$ & $59 \pm 8$ & $55 \pm 11$ & $63 \pm 9$ & $<0.0001$ \\
\hline$A R \geq 2, n(\%)$ & $123(35 \%)$ & $9(18 \%)$ & $1(2 \%)$ & $<0.0001$ \\
\hline \multicolumn{5}{|l|}{ Aorta diameter at baseline } \\
\hline Aortic annulus, mm & $25.0 \pm 3$ & $24.6 \pm 3$ & $24.6 \pm 2$ & 0.49 \\
\hline Sinuses of Valsalva, mm & $37.1 \pm 6$ & $41.5 \pm 6$ & $41.8 \pm 6$ & $<0.0001^{*} \dagger$ \\
\hline Sinotubular junction, mm & $31.5 \pm 5$ & $37.5 \pm 5$ & $33.3 \pm 5$ & $<0.0001^{*} \dagger \ddagger$ \\
\hline Ascending aorta, mm & $37.9 \pm 6$ & $44.5 \pm 4$ & $32.4 \pm 5$ & $<0.0001^{*} \dagger \ddagger$ \\
\hline \multicolumn{5}{|l|}{ Dilatation rate, $\mathrm{mm} / \mathrm{year}$} \\
\hline Aortic annulus & $0.05 \pm 0.2 \S$ & $0.005 \pm 0.2$ & $0.04 \pm 0.7$ & 0.51 \\
\hline Sinuses of Valsalva & $0.21 \pm 0.4 \S$ & $0.09 \pm 0.2 \S$ & $0.49 \pm 0.5 \S$ & $<0.0001^{*} \dagger \ddagger$ \\
\hline Sino-tubular junction & $0.18 \pm 0.5 \S$ & $0.10 \pm 0.2 \S$ & $0.10 \pm 1.2$ & 0.50 \\
\hline Ascending aorta & $0.42 \pm 0.6 \S$ & $0.20 \pm 0.3 \S$ & $0.12 \pm 1.0 \S$ & $0.0005^{*} \dagger$ \\
\hline Maximal dilatation rate & $0.42 \pm 0.6$ & $0.20 \pm 0.3$ & $0.49 \pm 0.5$ & $0.02 * \ddagger$ \\
\hline \multicolumn{5}{|c|}{$\begin{array}{l}\text { *Indicates significant differences between BAV and DA. } \\
\text { tIndicates significant differences between BAV and MFS. } \\
\text { fIndicates significant differences between MFS and DA. } \\
\text { §Indicates significant differences between baseline and follow-up diameters. } \\
\text { Larger baseline aortic diameters in DA patients reflect the inclusion criteria of } 40 \mathrm{~mm} \text { for these patients versus } 37 \mathrm{~mm} \text { for BAV and MFS. } \\
\text { AR, aortic regurgitation; BAV, bicuspid aortic valve; BP, blood pressure; DA, degenerative aortopathy; MFS, Marfan syndrome. }\end{array}$} \\
\hline
\end{tabular}

aortic phenotype at baseline was predominant AA dilatation. Patients with non-dilated aorta (normal shape) were younger with a smaller BSA compared to other aortic patterns, and the four types of BAV were equally distributed (figure 2B). Patients with predominant Valsalva sinus dilatation were more frequently men and had predominantly a typical BAV (figure 2C).

In multivariate analysis, independent determinants of Valsalva $>$ AA aortic phenotype were younger age $(p=0.0001)$, male gender $(p<0.0001)$, higher BSA $(p=0.002)$, and the typical morphology of BAV $(p=0.0001)$. AR $\geq$ moderate was not an independent determinant of the Valsalva $>A A$ aortic phenotype.

Aortic diameters according to BAV subtypes are summarised in table 3. Independent predictors of aortic diameters at baseline were older age, male gender,higher BSA for all levels of the aorta, and the presence of $\mathrm{AR} \geq$ moderate only at the level of the annulus. On multivariate analyses, after adjustment for age, gender, BSA,
Figure 2 Distribution of the type of bicuspid aortic valve (BAV). Panel A: entire population of BAV (grey colour denotes indeterminate type). Panel $\mathrm{B}$ : normal aortic shape without dilatation. Panel C: predominant aortic dilatation at the level of the Valsalva sinuses. Panel D: predominant dilatation at the level of tubular ascending aorta above the sinotubular junction. Access the article online to view this figure in colour.
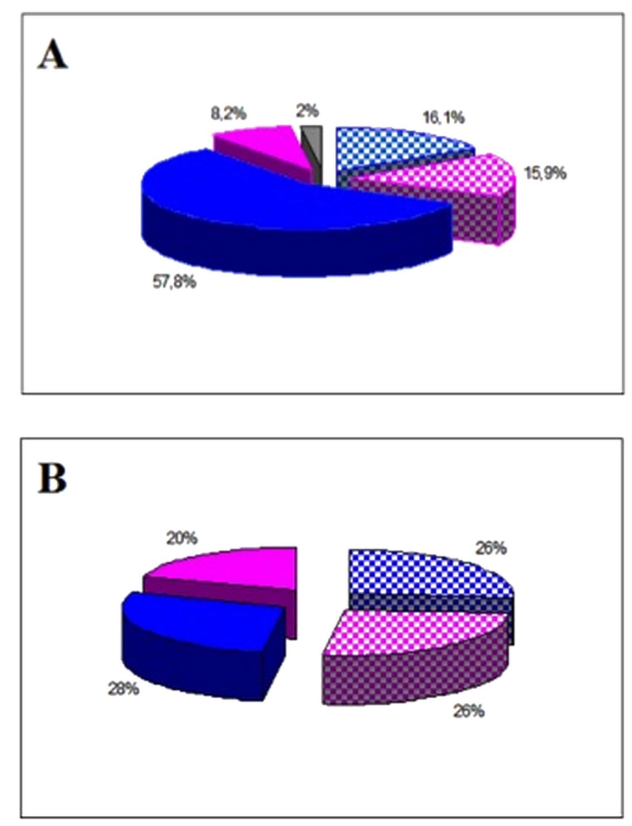

Typical with raphé

Q Typical without raphé

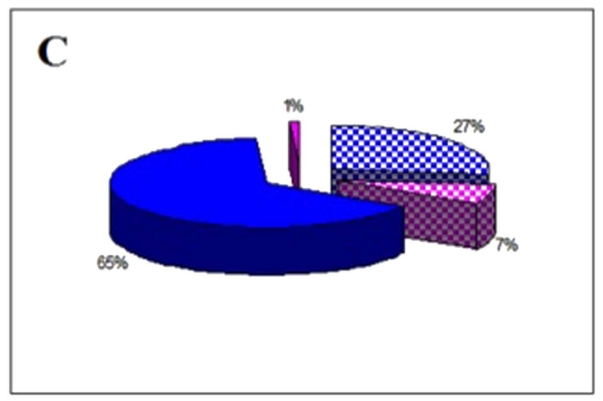

D

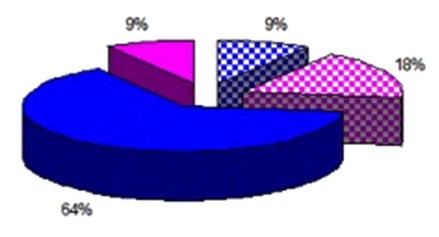

Atypical with raphé

Q Atypical without raphé 
Table 2 Baseline characteristics of BAV patients according to aortic phenotype

\begin{tabular}{|c|c|c|c|c|}
\hline & $\begin{array}{l}\text { No aortic dilatation, } \\
\text { normal shape } \\
N=47\end{array}$ & $\begin{array}{l}\text { Aortic dilatation } \\
\text { Valsalva }>\mathrm{AA} \\
\mathrm{N}=93\end{array}$ & $\begin{array}{l}\text { Aortic dilatation } \\
\mathrm{AA}>\text { Valsalva } \\
\mathrm{N}=213\end{array}$ & $\mathrm{p}$ Value \\
\hline \multicolumn{5}{|l|}{ Clinical characteristics } \\
\hline Age (years) & $38 \pm 16$ & $49 \pm 14$ & $50 \pm 14$ & $<0.0001^{*}$ \\
\hline Male gender $\mathrm{n}(\%)$ & $33(70 \%)$ & $84(90 \%)$ & $134(65 \%)$ & $<0.004 \dagger$ \\
\hline $\mathrm{BSA}\left(\mathrm{m}^{2}\right)$ & $1.88 \pm 0.21$ & $2.0 \pm 0.20$ & $2.0 \pm 0.24$ & $0.006^{*}$ \\
\hline \multicolumn{5}{|c|}{ Echocardiographic characteristics } \\
\hline $\mathrm{AR} \geq$ moderate & $13(28 \%)$ & $44(47 \%)$ & $65(31 \%)$ & $0.01 \dagger$ \\
\hline $\mathrm{AS} \geq$ moderate & $4(9 \%)$ & $9(12 \%)$ & $37(19 \%)$ & 0.15 \\
\hline \multicolumn{5}{|l|}{ BAV morphology } \\
\hline Typical & $25(54 \%)$ & $83(92 \%)$ & $150(72 \%)$ & $<0.0001 \ddagger$ \\
\hline All types (\%) & & & & $<0.0001 \ddagger$ \\
\hline Typical with raphe & 28 & 86 & 64 & \\
\hline Typical without raphe & 26 & 27 & 9 & \\
\hline Atypical with raphe & 20 & 1 & 9 & \\
\hline Atypical without raphe & 26 & 6 & 18 & \\
\hline
\end{tabular}

*NS between Valsalva $>A A$ and $A A>V a l s a l v a$.

tNS between $A A>V a l s a l v a$ and normal shape.

¥Significant for predominance of typical type in Valsalva>AA phenotype.

$A A$, ascending aorta; $A S$, aortic stenosis; BAV, bicuspid aortic valve.

$\mathrm{AR} \geq$ moderate, and heart rate, the typical type of $\mathrm{BAV}$ remained an independent predictor of larger aortic diameters at the level of the annulus $(p=0.009)$, Valsalva sinuses $(p<0.0001)$, STJ $(p=0.04)$, but not at the AA level $(\mathrm{p}=0.15)$.

Comparison with other aortopathies

Comparing BAV to MFS and DA (table 1), baseline aortic annulus diameters were similar. However, BAV had smaller Valsalva sinuses versus MFS and DA, and the AA was largest in DA versus BAV and
MFS. Thus, the phenotype of aortic dilatation was different, involving all segments in DA, involving preferentially Valsalva sinuses in MFS, and preferentially the AA in BAV. However, despite the preferential AA dilatation in BAV (60\% of BAV patients at baseline), $27 \%$ had predominant dilatation of the Valsalva sinuses (table 2).

\section{Aortic dilatation rates in BAV patients}

$\mathrm{BAV}$ aortic dilatation rates per year, level and group are indicated in the lower third of table 1. During follow-up, aortic diameters

Table 3 Baseline characteristics and yearly aortic dilatation rate according to BAV subtype

\begin{tabular}{|c|c|c|c|c|c|}
\hline & $\begin{array}{l}\text { Typical, } \\
\text { no raphe } \\
\mathrm{N}=57\end{array}$ & $\begin{array}{l}\text { Atypical, } \\
\text { no raphe } \\
\mathrm{N}=56\end{array}$ & $\begin{array}{l}\text { Typical, } \\
\text { with raphe } \\
\mathrm{N}=204\end{array}$ & $\begin{array}{l}\text { Atypical, } \\
\text { with raphe } \\
\mathrm{N}=29\end{array}$ & $\mathrm{p}$ Value \\
\hline \multicolumn{6}{|l|}{ Clinical characteristics } \\
\hline Age, years & $43 \pm 15$ & $46 \pm 15$ & $50 \pm 14$ & $48 \pm 15$ & 0.004 \\
\hline $\begin{array}{l}\text { Male gender, } \mathrm{n} \\
(\%)\end{array}$ & $41(72 \%)$ & $34(61 \%)$ & $155(76 \%)$ & $20(69 \%)$ & 0.15 \\
\hline \multicolumn{6}{|c|}{ Echocardiographic characteristics } \\
\hline$\%$ Ejection fraction, & $59.4 \pm 8$ & $57.2 \pm 8$ & 59.9 & 61.3 & 0.64 \\
\hline$A R \geq$ moderate & $20(35 \%)$ & $11(20 \%)$ & $84(41 \%)$ & $8(28 \%)$ & $0.02^{*}$ \\
\hline $\mathrm{AS} \geq$ moderate & $6(12 \%)$ & $10(20 \%)$ & $30(16 \%)$ & $4(15 \%)$ & 0.69 \\
\hline \multicolumn{6}{|c|}{ Baseline aortic dimension } \\
\hline Annulus & $25 \pm 3.6$ & $25 \pm 3.5$ & $25 \pm 2.9$ & $25 \pm 1.9$ & 0.77 \\
\hline Valsalva sinuses & $37.7 \pm 5.7$ & $34.7 \pm 5.7$ & $38.1 \pm 5.4$ & $34.1 \pm 3.3$ & $0.0001 *$ \\
\hline $\begin{array}{l}\text { Sinotubular } \\
\text { junction }\end{array}$ & $31.1 \pm 5.2$ & $31.1 \pm 5.2$ & $32.2 \pm 5.3$ & $29.1 \pm 3.6$ & $0.02 \dagger$ \\
\hline Ascending aorta & $36.5 \pm 6.3$ & $36.8 \pm 7.5$ & $39.1 \pm 6.1$ & $35.8 \pm 6.5$ & $0.005 \ddagger$ \\
\hline \multicolumn{6}{|l|}{ Yearly rate, mm/year } \\
\hline Annulus & $0.05 \pm 0.3$ & $0.02 \pm 0.1$ & $0.06 \pm 0.2$ & $0.05 \pm 0.1$ & 0.49 \\
\hline Valsalva sinuses & $0.24 \pm 0.3$ & $0.25 \pm 0.5$ & $0.19 \pm 0.3$ & $0.20 \pm 0.4$ & 0.61 \\
\hline $\begin{array}{l}\text { Sinotubular } \\
\text { junction }\end{array}$ & $0.24 \pm 0.6$ & $0.12 \pm 0.4$ & $0.21 \pm 0.5$ & $0.05 \pm 0.3$ & 0.29 \\
\hline Ascending aorta & $0.39 \pm 0.5$ & $0.51 \pm 0.8$ & $0.40 \pm 0.5$ & $0.33 \pm 0.6$ & 0.54 \\
\hline
\end{tabular}


significantly increased at all levels in patients with BAV (all $\mathrm{p}<0.001$, table 1 ) and yearly aortic dilatation rate increased from the aortic annulus to the AA (from $0.05 \pm 0.2 \mathrm{~mm} /$ year to $0.42 \pm 0.6 \mathrm{~mm} /$ year, respectively). The maximum dilation rate was $0.42 \pm 0.6 \mathrm{~mm} /$ year (median $0.3 \mathrm{~mm} /$ year, $25-75$ th centile $0-0.64$ ) at the AA level. These dilatation rates were greater than expected yearly dilatation rates derived from Roman's nomograms $(0.08 \pm 0.3 \mathrm{~mm} /$ year for Valsalva sinuses $(p<0.0001)$, and $0.07 \pm 0.2 \mathrm{~mm} /$ year for STJ $(\mathrm{p}=0.005))$. During follow-up, prescribed treatment was ACE inhibitors in $25 \%$ of cases, angiotensin receptor blockers in $7 \%$, and $\beta$-blockers in $32 \%$. There was no independent association of the treatment with lower aortic dilatation progression rate (all $\mathrm{p}>0.13$ ).

Analysing determinants of aortic dilatation rate in BAV, the range (CI $10 \%$ to $90 \%$ ) of dilatation rate was wide and increased from the annulus $(0.3 \mathrm{~mm})$ to Valsalva sinuses $(0.6 \mathrm{~mm})$, to the AA $(1.0 \mathrm{~mm})$. Baseline characteristics associated with greater maximum BAV aortic dilatation rate (any aortic level) were younger age and smaller aortic diameter at baseline; there was no association between aortic dilatation rate and gender, BSA, blood pressure, heart rate, aortic stenosis/ regurgitation at baseline, pattern of aortic dilatation, or type of BAV. The aortic dilatation rate in subgroups of BAV was unrelated to morphology or the presence of raphe (table 3). We also evaluated predictors of progression for each segment. At the level of the annulus, no baseline parameter was associated with rapid progression. For Valsalva sinuses, rapid progression was associated with younger age (OR per year: 0.97 95\% CI $(0.96$ to 0.99$), p=0.007)$ and with the degree of $A R$ at diagnosis (OR per mm of vena contracta: 1.1 (95\% CI 1.01 to 1.3$), p=0.03)$. For STJ, rapid progression was associated with smaller STJ diameters at baseline (OR per mm: 0.94 (95\% CI 0.89 to 0.99), $\mathrm{p}=0.02$ ) and tended to be associated with younger age (OR per year: 0.98 (0.97 to 1.00$), \mathrm{p}=0.05)$. For $\mathrm{AA}$, rapid progression was associated with lower AA diameter at baseline (OR per mm: 0.95 (95\% CI 0.91 to 0.98 ), $\mathrm{p}=0.008$ ) and tended to be associated with younger age (OR per year and 0.98 (95\% CI 0.97 to 1.00$), p=0.05)$. Forcing the type of BAV into the model did not affect the results or show a morphology-dilatation rate link. Importantly, non-progressors could not be identified from baseline aortic diameter, measured at the level of the sinuses of Valsalva $(p=0.34)$ or STJ $(p=0.23)$. Above the STJ, nonprogressors had greater baseline aortic diameter $(p=0.03)$.

\section{Comparison with other aortopathies}

Contrasting aortic dilatation progression in BAV versus MFS versus DA, dilatation rates did not differ at the annulus level $(p=0.51)$ and the STJ level $(p=0.57)$. In contrast, the largest aortic dilatation rate was observed in MFS at the level of the Valsalva sinuses $(0.49 \pm 0.5 \mathrm{~mm} /$ year (median $0.42 \mathrm{~mm} /$ year, $25-$ 75 th centile $0.13-0.8)$ ) and in BAV at the AA level (both $\mathrm{p}<0.03$, table 1). Although occurring at different levels, these maximum aortic dilatation rates in MFS and BAV did not differ in mean magnitude and were greater than the maximum aortic dilatation rate in DA at any level $(\mathrm{p}=0.02)$. However, despite similar mean progression of magnitude, the distribution of maximal aortic dilatation rate differed between patients with BAV and MFS (figure 3) and a larger percentage of patients with BAV $(43 \%$ vs $20 \%, \mathrm{p}=0.01)$ did not incur aortic dilatation during follow-up. At last follow-up, comparing aortic dimensions in BAV, MFS and DA, the aortic annulus was similar in all groups $(p=0.40)$ and the Valsalva sinuses $(38 \pm 6,44 \pm 6$ and $41.8 \pm 6 \mathrm{~mm}$ ) were different between all groups (all $\mathrm{p}<0.05)$, being largest in MFS and smallest in BAV. The STJ $(32 \pm 5,35$ \pm 7 , and $37.5 \pm 6 \mathrm{~mm}$ ) was also different between all groups (all $\mathrm{p}<0.05)$, but largest in DA and smallest in BAV. The AA $(39 \pm 6$, $34 \pm 7$, and $45 \pm 4 \mathrm{~mm}$ ) was also different between all groups (all $\mathrm{p}<0.05$ ), but largest in DA and smallest in MFS. Thus, the phenotype of Valsalva sinuses $>$ AA was present in $96 \%$ of MFS cases vs $30 \%$ of DA and $36 \%$ of BAV $(p<0.0001)$.

\section{DISCUSSION}

To our knowledge, this collaborative tertiary referral USEuropean registry is the largest echocardiographic study evaluating valvulo-aortic phenotypes, rates, and patterns of aortic dilatation and their determinants in BAV patients. In addition, we compared for the first time progression in BAV to that of other aortopathies (MFS and DA) in patients of similar gender, systolic blood pressure, and minimum interval to follow-up. The most important finding of this study is that in patients with BAV, the fastest aortic dilatation rate is observed at the AA level $(0.42 \mathrm{~mm} /$ year $)$, is independent of $\mathrm{BAV}$ morphology, and is similar to the aortic dilatation rate observed in MFS patients at the level of the Valsalva sinuses. Secondly, the AA dilatation rate is heterogeneous in BAV patients, with significantly more patients characterised as 'non-progressors' compared to MFS. These different phenotypic characteristics and different progression patterns could be partially linked to the relatively low risk of aortic catastrophes in patients with $\mathrm{BAV}^{5}$ Thirdly, in patients with BAV, a normal aortic diameter at baseline does not predict a lower rate of dilatation (and vice versa), and determinants of rapid progression are lower age and smaller baseline aortic diameter, such that systematic aortic size follow-up is warranted in all patients with BAV, regardless of baseline measurement. This also has important research implications-trials of medical therapy directed at decreasing dilatation rates would be most powerful when applied to younger patients with smaller baseline aortas. Fourthly, all BAV types can exhibit a non-dilated aorta but the most common site of dilation for all BAV types is the AA. Nevertheless, we corroborate the presence of a group of BAV patients with predominant dilatation of Valsalva sinuses, ${ }^{16}{ }^{24}$ characterised by a preponderance of typical BAV morphology and male gender. We expand knowledge within this notion by observing that a typical BAV independently predicts baseline dilatation of the entire proximal aorta (annulus, sinuses, STJ), except AA. Finally, for the time interval studied, we show that BAV morphology is not associated with aortic dilatation progression rates.

\section{BAV and the aorta}

In patients with $\mathrm{BAV}$, we observed three basic aortic phenotypes at baseline: (1) a normal shape, which was more common in younger patients; (2) a predominance of Valsalva sinus dilatation, which was observed mostly in males with typical BAV; (3) a predominance of AA dilatation, which was independent of BAV morphology and was the most common pattern of aortic dilatation. Our large study reconciles the discordances between previously described predominant patterns of aortic dilatation in $\mathrm{BAV}^{6}{ }^{7}{ }^{13-16}$ and by showing not just a predominance of AA dilatation but different valvulo-aortic phenotypes, supports a genetically heterogeneous origin for BAV with dissimilar embryology. ${ }^{25}$ The predominant Valsalva sinus dilatation in BAV or 'sinuses phenotype' was first described by Della Corte et $a,^{26}$ and the variable prevalence between their study and ours likely relates to different inclusion definitions. 
Figure 3 Comparative distribution of the aortic dilatation rate in the segment of the aorta more prone to dilatation. Tubular ascending aorta for patients with bicuspid aortic valve (BAV) and sinuses of Valsalva in patients with Marfan syndrome. Although the mean value is similar ( 0.42 and $0.49 \mathrm{~mm} /$ year respectively), the distribution of the population is very different.
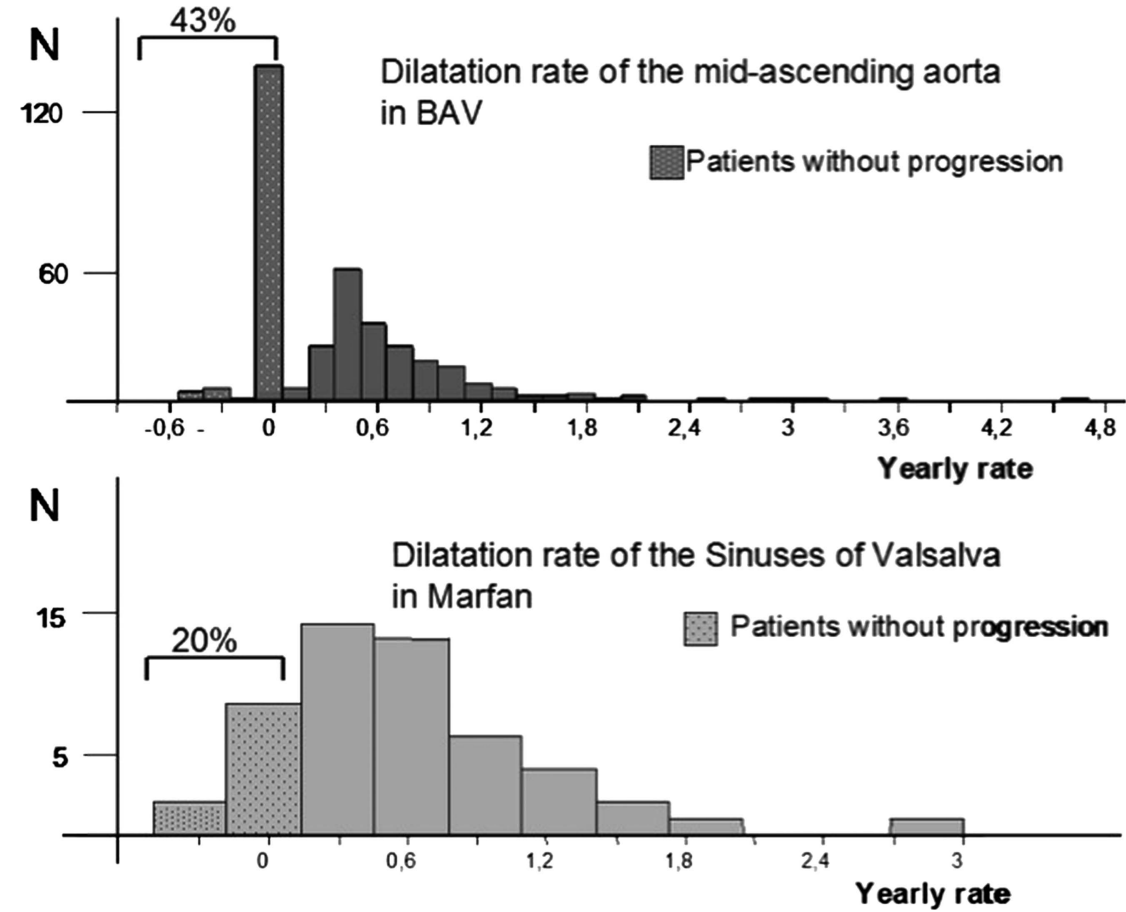

\section{Aortic dilatation rates in BAV}

In BAV patients, the aortic dilatation rate was maximal at the AA level $-0.42 \mathrm{~mm} / \mathrm{year}$ (two times greater than at the level of the sinuses of Valsalva) - and could not be predicted by the BAV type. In addition, the presence of aortic dilatation at baseline was not a risk factor for rapid aortic dilatation at any aortic level and younger patients tended to dilate more rapidly than older patients. These findings may seem to contradict previous reports in which older age and baseline aortic dilatation were predictors of aneurysm formation. ${ }^{35}$ The likely explanation for this discrepancy is that the current study evaluated intermediate term dilatation rates (within $3.6 \pm 1.2$ years) while prior studies evaluated long term end points (ie, aneurysm formation) and not dilatation rates. Indeed, most patients with a dilated aorta at baseline were also older in the current study.

Regarding factors associated with aortic dilatation rates, both typical $\mathrm{BAV}^{15}$ and atypical $\mathrm{BAV}^{27}$ have been suggested as predictors of faster aortic dilatation. Our large study is adequately powered to address this issue and could not detect a link between the BAV type and the rate of aortic dilatation. Furthermore, within each type of BAV, the aortic dilatation rate displayed a wide range, supporting the fact that risk stratification for aortic dilatation rate based on BAV morphology would be inadequate. We found no association between BAV dysfunction and aortic dilatation progression rates (except AR and Valsalva sinuses). Aortic stenosis has been linked to aneurysm formation, ${ }^{5} 28$ as a long term outcome. It is possible that aortic stenosis is a marker of increased age, which in turn is related to development of aneurysms. In addition, there was a paucity $(14 \%)$ of significant stenosis in our BAV group. Although this paucity of stenosis favours the 'histological aortic weakness' concept in our study, there is clear evidence that non-stenotic $\mathrm{BAVs}$ are nonetheless intrinsically dysfunctional, generating significant flow abnormalities that increase systolic shear stress in the aorta. ${ }^{29}$ We hypothesise that both haemodynamic and histological aspects are important. Moreover, it is likely that other yet unknown genetic and environmental factors are at play. Mean aortic dilatation rates were considerable in earlier reports, with values ranging from $0.8-1.2 \mathrm{~mm} / \mathrm{year},{ }^{11} 1314$ whereas we found a rate of $0.42 \mathrm{~mm} / \mathrm{year}$ in our report, which is very similar to the $0.37 \mathrm{~mm} /$ year in the recent report from Thanassoulis et al. ${ }^{15}$ Our interpretation of this difference is that improved measurement accuracy with enhanced imaging and more widespread use of systematic follow-up imaging by echocardiography yield more realistic progression rates in the recent era.

\section{Comparison with other aortopathies}

The mean aortic dilatation rate in BAV patients was greater compared to that observed in DA patients, and similar to that observed in patients with MFS carrying an FBN1 mutation. Indeed, BAV and MFS aortas display similar histological features, so that it may be impossible to recognise the aetiology of aneurysms of the aorta based on histology alone. However, several differences between MFS and BAV can be emphasised. The maximal aortic dilatation rate is observed above the STJ in the population with BAV, whereas it is observed at the level of the Valsalva sinuses in the MFS population, suggesting different anatomic sites of aortic vulnerability. In addition, although mean aortic dilatation rates are similar in MFS and BAV, almost half of the BAV patients are non-progressors, whereas the majority of patients with MFS progress over time. In our study, AR was more frequent with BAV than with MFS or DA, despite larger sinuses of Valsalva in these latter aortopathies. Thus, AR in BAV is possibly a consequence of the abnormal valve rather than proximal aortic dilatation. Nevertheless, AR was associated with dilatation progression at the Valsalva sinuses level.

\section{Study limitations and strengths}

The design of our study is retrospective-observational and may be prone to bias. However, the large size of our study and prospective aortic measurement undertaken by an experienced observer (DD), with blinding of the aortic measurement to any clinical or outcome data, suggest the likelihood of bias is low. Furthermore, matching of groups (BAV, MFS, DA) in regard to factors that may affect aortic dilatation over time also minimises 
the possibility of inter-group bias. However, baseline aortic diameter inclusion (larger for DA) and age difference (older for DA) could partially explain the maximum aortic dilatation rates in DA patients being smaller than in MFS and BAV. Also, we used actual blood pressure measurement and not 'history of hypertension' in our definitions, and the DA group likely has a higher prevalence of hypertension.

Obviously, we could not correct for age as different aortopathies are clinically recognised at variable ages of the patients. Age is likely a determinant of dilatation progression and thus limits the quantitative comparison between groups. Importantly, when matching patients with BAV and MFS for ages between 30-50 years (BAV 41.5 \pm 6 , MFS 40.2 $\pm 6 ; p=0.27$ ), we observed no difference in maximal progression rate $(\mathrm{p}=0.5)$ and corroborated faster progression of sinuses for MFS and tubular ascending for $\mathrm{BAV}(\mathrm{p}<0.05)$. Furthermore, the age groups are representative of current clinical practice for each aortopathy, therefore their quantitative comparison seems fair from the clinical perspective. Aortic measurements were performed by echocardiography which was consistently performed at baseline and follow-up, whereas CT or MRI imaging in accordance with current recommendations ${ }^{30}$ was not consistently performed. Nevertheless, echocardiographic measurements remain the current standard of care $^{30}$ and in patients with concomitant MRI there was no detectable difference between techniques. Notwithstanding, analysis of aortic arch dimensions was not possible in this study. The results of this study are applicable only to adults ( $\geq 18$ years) within a tertiary referral based practice, and are not meant to represent the entire spectrum of MFS and DA, as these were matched by gender to patients with BAV. The conclusions of our study represent the observations made over a 25-75 centile interval time of $2.7-4.5$ years and longer follow-up studies should be performed. Finally, the use of annualised aortic dilatation rates and lack of an intermediate measurement between the first and follow-up echocardiograms limit the analysis of more complex aortic growth attributes such as potential time dependent growth decelerations.

\section{Clinical/research implications}

In BAV patients, baseline AA dilatation is most common and independent of valve morphology, while baseline sinus of Valsalva dilatation is associated with typical BAV morphology and male gender. The aortic dilatation rate is maximal at the AA, independent of valve function and morphology, and similar but not greater than that observed at the sinuses of Valsalva in patients with MFS. Thus, dilatation above the STJ should be closely monitored in BAV patients. The observed dilatation rates cannot be inferred from BAV morphology or baseline aortic diameter measured once, and therefore systematic follow-up is warranted when a BAV is recognised. Furthermore, because the aortic dilatation rate may significantly differ between individual patients, only specific follow-up measurements can determine individual size-dependent aortic risk. In addition, although there is an independent association between typical BAV and the predominant Valsalva sinus phenotype, the risk of dilatation and aortic surveillance should not be based on BAV morphology. Aortic dilatation rates at the level of the sinuses of Valsalva are significantly less prominent than AA, supporting a recent report $^{31}$ which indicates that preserving the Valsalva sinuses at the time of AA replacement in patients with BAV and no significant dilatation of the Valsalva sinuses, was not associated with reintervention due to progressive dilatation of Valsalva sinuses at follow-up. Trials of medical therapy directed at decreasing dilatation rates would be most revealing when applied to younger patients with smaller baseline aortas. Lastly, the dilatation rate heterogeneity and its independence from BAV morphology suggest a higher level of complexity in genetic and environmental determinants of BAV aortopathy, which need to be determined.

\section{Key messages}

What is already known about this subject

Bicuspid aortic valve (BAV) is associated with aortic dilatation. Patterns of dilatation are not well defined and comparison with other aortopathies is lacking.

What does this study add

In BAV, tubular ascending aorta dilatation is the most common pattern and exhibits the fastest growing rate, irrespective of valve morphology and function. Sinuses growth rate is slower. Baseline aortic diameter does not proportionally predict the progression rate. Aortic dilatation progresses equally fast in BAV and Marfan syndrome, but a significantly higher BAV patient proportion does not progress at all.

How might this impact on clinical practice Dilatation above the sinotubular junction should be closely monitored in BAV patients. The risk of dilatation and aortic surveillance should not be based on BAV morphology. Systematic follow-up is warranted when a BAV is recognised. Surgical preservation of a normal aortic sinuses appears theoretically reasonable. Aortic dilatation patterns are different between BAV and Marfan syndrome patients.

Contributors All authors contributed to: conception and design, acquisition of data or analysis and interpretation of data; drafting the article or revising it critically for important intellectual content; and final approval of the version published.

Funding PHRC AOM09093 (GJ), ANR 2010 BLAN 1129 from the French National Research Agency (GJ).

\section{Competing interests None.}

Ethics approval Institutional Review Boards of participating centres.

Provenance and peer review Not commissioned; externally peer reviewed.

\section{REFERENCES}

1 Roger VL, Go AS, Lloyd-Jones DM, et al. Heart disease and stroke statistics-2011 update: a report from the American Heart Association. Circulation 2011;123: e18-209.

2 Lamas CC, Eykyn SJ. Bicuspid aortic valve-a silent danger: analysis of 50 cases of infective endocarditis. Clin Infect Dis 2000;30:336-41.

3 Michelena HI, Desjardins VA, Avierinos JF, et al. Natural history of asymptomatic patients with normally functioning or minimally dysfunctional bicuspid aortic valve in the community. Circulation 2008;117:2776-84

4 Tzemos N, Therrien J, Yip J, et al. Outcomes in adults with bicuspid aortic valves. JAMA 2008;300:1317-25.

5 Michelena $\mathrm{HI}$, Khanna $A D$, Mahoney $\mathrm{D}$, et al. Incidence of aortic complications in patients with bicuspid aortic valves. JAMA 2011;306:1104-12.

6 Keane MG, Wiegers SE, Plappert T, et al. Bicuspid aortic valves are associated with aortic dilatation out of proportion to coexistent valvular lesions. Circulation 2000;102:III35-9.

7 Nkomo VT, Enriquez-Sarano M, Ammash NM, et al. Bicuspid aortic valve associated with aortic dilatation: a community-based study. Arterioscler Thromb Vasc Biol 2003; $23: 351-6$

8 Fedak PW, Verma S, David TE, et al. Clinical and pathophysiological implications of a bicuspid aortic valve. Circulation 2002:106:900-4.

9 Nataatmadja M, West $M$, West J, et al. Abnormal extracellular matrix protein transport associated with increased apoptosis of vascular smooth muscle cells in 
Marfan syndrome and bicuspid aortic valve thoracic aortic aneurysm. Circulation 2003;108:II329-34.

10 Bonow RO, Carabello BA, Chatterjee K, et al. ACC/AHA 2006 guidelines for the management of patients with valvular heart disease: a report of the American College of Cardiology/American Heart Association Task Force on Practice Guidelines (writing Committee to Revise the 1998 guidelines for the management of patients with valvular heart disease) developed in collaboration with the Society of Cardiovascular Anesthesiologists endorsed by the Society for Cardiovascular Angiography and Interventions and the Society of Thoracic Surgeons. J Am Coll Cardiol 2006;48:e1-148.

11 Beroukhim RS, Kruzick TL, Taylor AL, et al. Progression of aortic dilation in children with a functionally normal bicuspid aortic valve. Am J Cardiol 2006;98:828-30.

12 Warren $\mathrm{AE}$, Boyd ML, O'Connell C, et al. Dilatation of the ascending aorta in paediatric patients with bicuspid aortic valve: frequency, rate of progression and risk factors. Heart 2006;92:1496-500.

13 Ferencik M, Pape LA. Changes in size of ascending aorta and aortic valve function with time in patients with congenitally bicuspid aortic valves. Am J Cardiol 2003;92:43-6.

14 La Canna G, Ficarra E, Tsagalau E, et al. Progression rate of ascending aortic dilation in patients with normally functioning bicuspid and tricuspid aortic valves. Am J Cardiol 2006;98:249-53.

15 Thanassoulis G, Yip JW, Filion K, et al. Retrospective study to identify predictors of the presence and rapid progression of aortic dilatation in patients with bicuspid aortic valves. Nat Clin Pract Cardiovasc Med 2008;5:821-8.

16 Schaefer BM, Lewin MB, Stout KK, et al. The bicuspid aortic valve: an integrated phenotypic classification of leaflet morphology and aortic root shape. Heart 2008;94:1634-8.

17 Svensson LG, Kim KH, Lytle BW, et al. Relationship of aortic cross-sectional area to height ratio and the risk of aortic dissection in patients with bicuspid aortic valves. J Thorac Cardiovasc Surg 2003;126:892-3.

18 De Paepe A, Devereux RB, Dietz HC, et al. Revised diagnostic criteria for the Marfan syndrome. Am J Med Genet 1996;62:417-26.

19 Quinones M, Waggoner A, Reduto L. A new, simplified and accurate method for determining ejection fraction with two-dimensional echocardiography. Circulation 1981:64:744-53.

20 Rich S, Sheikh A, Gallastegui J, et al. Determination of left ventricular ejection fraction by visual estimation during real-time two-dimensional echocardiography. Am Heart J 1982;104:603-6.
21 Zoghbi WA, Enriquez-Sarano M, Foster E, et al. Recommendations for evaluation of the severity of native valvular regurgitation with two-dimensional and Doppler echocardiography. J Am Soc Echocardiogr 2003;16:777-802.

22 Lang RM, Bierig M, Devereux RB, et al. Recommendations for chamber quantification: a report from the American Society of Echocardiography's Guidelines and Standards Committee and the Chamber Quantification Writing Group, developed in conjunction with the European Association of Echocardiography, a branch of the European Society of Cardiology. J Am Soc Echocardiogr 2005;18:1440-63.

23 Roman MJ, Devereux RB, Kramer-Fox R, et al. Two-dimensional echocardiographic aortic root dimensions in normal children and adults. Am J Cardiol 1989;64:507-12.

24 Khoo C, Cheung C, Jue J. Patterns of aortic dilatation in bicuspid aortic valve-associated aortopathy. J Am Soc Echocardiogr 2013;26:600-5.

25 Fernandez B, Duran AC, Fernandez-Gallego T, et al. Bicuspid aortic valves with different spatial orientations of the leaflets are distinct etiological entities. J Am Coll Cardiol 2009;54:2312-18

26 Della Corte A, Bancone C, Quarto C, et al. Predictors of ascending aortic dilatation with bicuspid aortic valve: a wide spectrum of disease expression. Eur $\mathrm{J}$ Cardiothorac Surg 2007;31:397-404; discussion-5.

27 Holmes KW, Lehmann CU, Dalal D, et al. Progressive dilation of the ascending aorta in children with isolated bicuspid aortic valve. Am J Cardiol 2007;99:978-83.

28 Kim YG, Sun BJ, Park GM, et al. Aortopathy and bicuspid aortic valve: haemodynamic burden is main contributor to aortic dilatation. Heart 2012;98:1822-7.

29 Bissell MM, Hess AT, Biasiolli L, et al. Aortic dilation in bicuspid aortic valve disease: flow pattern is a major contributor and differs with valve fusion type. Circ Cardiovasc Imaging 2013;6:499-507.

30 Hiratzka LF, Bakris GL, Beckman JA, et al. 2010 ACCF/AHA/AATS/ACR/ASA/SCA/ SCAI/SIR/STS/SVM guidelines for the diagnosis and management of patients with thoracic aortic disease: a report of the American College of Cardiology Foundation/ American Heart Association Task Force on Practice Guidelines, American Association for Thoracic Surgery, American College of Radiology, American Stroke Association, Society of Cardiovascular Anesthesiologists, Society for Cardiovascular Angiography and Interventions, Society of Interventional Radiology, Society of Thoracic Surgeons, and Society for Vascular Medicine. Circulation 2010;121:e266-369.

31 Park CB, Greason KL, Suri RM, et al. Fate of nonreplaced sinuses of Valsalva in bicuspid aortic valve disease. J Thorac Cardiovasc Surg 2011;142:278-84. 